but a high specificity of $93 \%$ and could be useful to exclude rather than identify the presence of sputum eosinophilia.

Conclusion Combined testing using ACQ and BEos only identifies eosinophilia in 37\% and can't replace induced sputum, but may be useful to exclude sputum eosinophilia and prospective study is warranted.

\section{P50 DIFFICULT ASTHMA CLINICS: ARE THEY EFFECTIVE?}

I Macpherson, S Fielding, JG Douglas. Chest Clinic C, Aberdeen Royal Infirmary, Aberdeen, UK

\subsection{6/thoraxjnl-2014-206260.191}

Background "Difficult asthma" is defined as persistent symptoms and/or frequent exacerbations in patients with a diagnosis of asthma, despite treatment at step 4 or 5 of the BTS/SIGN guidelines ${ }^{1}$. "Difficult asthma" clinics have been set up in many UK hospitals, but there is no published evidence to suggest their effectiveness at reducing exacerbations or hospital admissions.

Aims To determine whether a regional difficult asthma clinic led to a reduction in prescription of oral steroid for exacerbations and a reduction in hospital admissions for asthma.

Methods A retrospective analysis of patients who attended this regional difficult asthma clinic between August 2009 and May 2013 was performed. Medical notes and GP letters were scrutinised to ascertain the number of hospital admissions two years before and two years after the first clinic appointment. For oral steroids, one year before and after was used. To compare these data Wilcoxon tests were used due to the skewed data.

Results For hospital admissions, data was available for 44 patients, mean (SD) age $44.2(14.1)$ and $66 \%$ female. The median (inter-quartile range (IQR)) number of hospital admissions in the two years prior to clinic was $2(0,3)$ compared to 0 $(0,1)$ in the two years following clinic visit $(p=0.014)$. Oral steroid prescription data was available for 54 patients, mean (SD) age 43.5 (14.4), 70\% female. The median (IQR) number of oral steroid courses required in the year prior to first clinic appointment was $6(3.75,10)$, which reduced to $2(0,4)$ in the year after first appointment $(\mathrm{p}<0.001)$.

Conclusion Our regional difficult asthma clinic significantly reduced both hospital admissions for asthma two years after first clinic appointment and number of oral steroid prescriptions one year after first clinic appointment. This study, although small, highlights the benefit of such clinics to patients and the potential for the reduction in use of NHS resources. They should be encouraged in all major centres.

\section{REFERENCE}

1 Prys-Picard CO, Campbell SM, Ayres JG, Miles JF, Niven RM. Consensus Difficult Asthma Consortium UK. Defining and investigating difficult asthma: developing quality indicators. Respiratory Medicine 2006;100(7):1254-61

\section{P51 BARRIERS AND FACILITATORS TO EFFECTIVE SELF- MANAGEMENT OF ASTHMA - A SYSTEMATIC REVIEW AND THEMATIC SYNTHESIS}

SE Kirby', C Miles ${ }^{2}$, E Arden-Close ${ }^{1}$, L Yardley ${ }^{2}$, A Bruton ${ }^{3}$, M Hankins ${ }^{4},{ }^{5}$ DM Thomas. ${ }^{1}$ Department of Psychology, University of Southampton, UK, NIHR Southampton Respiratory Biomedical Research Unit, UK; ${ }^{2}$ Department of Psychology, University of Southampton, UK; ${ }^{3}$ Faculty of Health Sciences, University of SouthamptonUK, NIHR Southampton Respiratory Biomedical Research Unit, UK; ${ }^{4}$ Real-World Evidence Solutions, IMS Health UK; ${ }^{5}$ Department of Primary Medical Care, University of Southampton, UK, NIHR Southampton Respiratory Biomedical Research Unit, UK

10.1136/thoraxjn-2014-206260.192
Introduction and objectives Self-management is an established approach to controlling asthma, recommended in guidelines. Despite the reported effectiveness of self-management, its promotion, uptake and use among patients, carers, and health-care professionals remain low. We conducted a systematic review and thematic synthesis of qualitative research into self-management in adults, children and adolescents with asthma. Our objective was to identify the perceived barriers and facilitators associated with reduced or improved effectiveness of asthma selfmanagement.

Method Electronic databases (Medline, EMBASE, AMED, CINAHL, and PsycINFO; 1996 - August 2013) and British Thoracic Society guidelines were searched for qualitative literature that explored factors relevant to facilitators and barriers to uptake, adherence, or outcomes of self-management in adults, children and adolescents with asthma. We assessed the methodological strengths of the studies using the Critical Appraisal Skills Programme (CASP) tool for qualitative studies, and conducted a thematic synthesis of included studies.

Results Of the 1532 studies initially identified, 34 papers were included in the review. Thematic synthesis identified 10 overarching themes (Figure 1) which suggest that barriers and facilitators of self-management included the perceived quality of the relationship between health-care professionals and patients, the perceived adequacy of education around self-management and medications, and positive and negative beliefs about asthma with regards to self-management and existing interventions. Self-management could also be helped or hindered by the amount and type of social support and perceived ease of access to healthcare. In addition, having a co-morbidity, mood/anxiety problems, and encountering professional barriers within the health care system (such as consultation time and access to lung function testing), are perceived to hinder successful self-management of asthma.

Conclusion Perceived barriers and facilitators occur at the level of individuals with asthma (and carers), health-care professionals, and organisations. These findings contribute to our understanding of why existing self-management interventions may not always be effective, and can be used to inform future study into areas where further intervention may improve the adoption of self-management of asthma. For example, future work could include addressing patient and carer beliefs in educational interventions and decisions involving treatment, or greater use of pharmacist educators, patient advocates, and technological interventions.

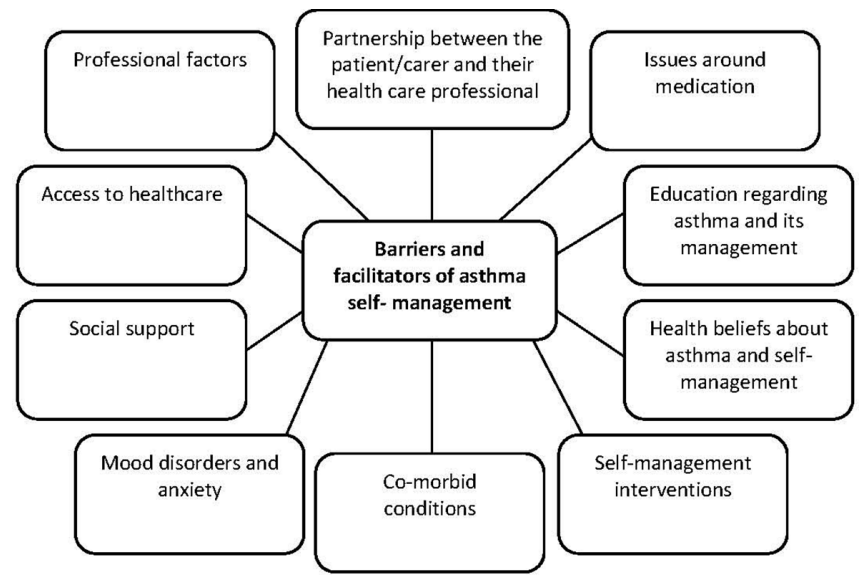

Abstract P51 Figure 1 Overarching themes identified during thematic synthesis 